\title{
Strong-coupling expansion for the Hubbard model in arbitrary dimension using slave bosons
}

\author{
P. J. H. Denteneer* \\ Instituut-Lorentz, University of Leiden, P.O. Box 9506, 2300 RA Leiden, The Netherlands
}

(Received 20 November 1995)

\begin{abstract}
A strong-coupling expansion for the antiferromagnetic phase of the Hubbard model is derived in the framework of the slave-boson mean-field approximation. The expansion can be obtained in terms of moments of the density of states of freely hopping electrons on a lattice, which in turn are obtained for hypercubic lattices in arbitrary dimension. The expansion is given for the case of half-filling and for the energy up to fifth order in the ratio of hopping integral $t$ over on-site interaction $U$, but can straightforwardly be generalized to the non-half-filled case and be extended to higher orders in $t / U$. For the energy the expansion is found to have an accuracy of better than $1 \%$ for $U / t \geqslant 8$. A comparison is given with an earlier perturbation expansion based on the linear-spin-wave approximation and with a similar expansion based on the Hartree-Fock approximation. The case of an infinite number of spatial dimensions is discussed.
\end{abstract}

\section{INTRODUCTION}

If the physics of strongly correlated fermions is to be described by the Hubbard model, ${ }^{1}$ electronic structure calculations for the cuprates indicate that the relevant parameter regime is where the on-site interaction $U$ is comparable to or larger than the bandwidth $W$ of freely hopping electrons. ${ }^{2} \mathrm{~A}$ natural strategy to try to describe this regime is to approach it from the two limiting cases of weak coupling $(U \ll W)$ and strong coupling $(U \gg W)$. The simplest mean-field approximation for the Hubbard model, the Hartree-Fock approximation (HFA), is at first sight a weak-coupling approximation, although, with some care, a reasonable description of the strong-coupling regime can also be given. ${ }^{3-6}$ Strongcoupling approaches have mainly been devoted to the onedimensional case (see, e.g., Ref. 7) and often take the limit $U \rightarrow \infty{ }^{8}$ A somewhat more sophisticated mean-field approximation, namely, that based on the slave-boson formulation due to Kotliar and Ruckenstein, ${ }^{9}$ is in principle not restricted to weak or strong coupling. In fact, it was shown that this so-called slave-boson mean-field (SBMF) approximation is equivalent to the Gutzwiller approximation to the Gutzwiller wave function, ${ }^{10}$ the latter of which improves upon the HFA especially in the intermediate-coupling regime. This SBMF approximation then allows us to approach the interesting intermediate-coupling regime from the strong-coupling side. Such an approximate strong-coupling approach can be particularly helpful since more rigorous quantum Monte Carlo calculations become increasingly cumbersome for stronger coupling. ${ }^{11}$

In a previous work, ${ }^{12}$ we compared the HFA and SBMF approximations for various simple magnetic phases, as well as computed the effective hopping and spin stiffness for the two-dimensional (one-band) Hubbard model. In this paper, I derive a large- $U$ expansion within the SBMF approximation for the Hubbard model in arbitrary dimension. Here I restrict myself to the antiferromagnetic phase at half-filling, but the extension to doping the antiferromagnet with electrons or holes is straightforward (though tedious).

In Sec. II, the slave-boson mean-field approximation is briefly introduced, and the resulting set of integral equations for the antiferromagnetic phase is given. A large- $U$ expansion is derived in Sec. III in terms of moments of the density of states for freely hopping electrons on a lattice, and compared to earlier numerical and analytical work in the literature. In Sec. IV, the expansion is made explicit for hypercubic lattices of arbitrary dimension. Finally, I discuss the convergence of the series and possible extensions. The Appendix contains useful formulas for the moments of the above-mentioned density of states.

\section{SLAVE-BOSON MEAN-FIELD APPROXIMATION}

The Hubbard Hamiltonian is ${ }^{1}$

$$
\mathscr{H}_{h}=-t \sum_{j, \delta, \sigma} c_{j+\delta, \sigma}^{\dagger} c_{j \sigma}+U \sum_{j} n_{j \uparrow} n_{j \downarrow},
$$

where $c_{j \sigma}$ is the annihilation operator for an electron at site $j$ with spin $\sigma$. Neighboring sites of site $j$ are denoted by $j+\delta$. $t$ is the hopping integral, $U$ the on-site interaction between electrons of opposite spin, and $n_{j \sigma}=c_{j \sigma}^{\dagger} c_{j \sigma}$ is the occupation number operator. The slave-boson approach of Kotliar and Ruckenstein consists of introducing bosons for each of the (four) possible electron occupancies of a site. The electron creation and annihilation operators are then modified such that the (one) boson corresponding to the electron occupancy is always present at each site. In a functional-integral description of the Hamiltonian the slave-boson mean-field (SBMF) approximation is when the Bose fields are independent of (imaginary) time. Different phases can be considered by assuming different forms of the position dependence of the Bose fields. For more details see Refs. 9 and 12. The SBMF approximation is an improvement over the HartreeFock approximation since it takes some local correlations into account; in particular, the density of doubly occupied sites is an independent parameter to be optimized.

The set of equations describing the antiferromagnetic (AF) phase within the SBMF is given by ${ }^{12}$

$$
m_{s}=2 \bar{\lambda} \int_{\bar{\mu}}^{\infty} d \varepsilon \frac{\mathscr{N}(\varepsilon)}{\left(\varepsilon^{2}+\bar{\lambda}^{2}\right)^{1 / 2}},
$$




$$
\begin{gathered}
\bar{\lambda}=\frac{2}{q_{s}}\left[q_{s}\right]_{m_{s}} \int_{\bar{\mu}}^{\infty} d \varepsilon \frac{\mathscr{N}(\varepsilon) \varepsilon^{2}}{\left(\varepsilon^{2}+\bar{\lambda}^{-2}\right)^{1 / 2}}, \\
U=\frac{\left[q_{s}\right]_{d}}{d} \int_{\bar{\mu}}^{\infty} d \varepsilon \frac{\mathscr{N}(\varepsilon) \varepsilon^{2}}{\left(\varepsilon^{2}+\bar{\lambda}^{-2}\right)^{1 / 2}}, \\
n=2 \int_{\bar{\mu}}^{\infty} d \varepsilon \mathscr{N}(\varepsilon),
\end{gathered}
$$

where $\bar{\lambda}$ is an internal (renormalized gap) parameter; $\bar{\mu}$ an effective chemical potential; and the band renormalization $q_{s}$ is a function of density $n$, sublattice magnetization $m_{s}$, and $d$ ( $d^{2}$ is the density of doubly occupied sites), and is given by

$$
q_{s}\left(n, m_{s}, d\right)=z\left(n, m_{s}, d\right) z\left(n,-m_{s}, d\right),
$$

where

$$
\begin{aligned}
& z\left(n, m_{s}, d\right) \\
& =\frac{\sqrt{\left(1-n+d^{2}\right)\left(n+m_{s}-2 d^{2}\right)}+d \sqrt{n-m_{s}-2 d^{2}}}{\left[\left(n+m_{s}\right)\left(1-\frac{n+m_{s}}{2}\right)\right]^{1 / 2}} .
\end{aligned}
$$

$\left[q_{s}\right]_{\alpha}$ is the derivative of $q_{s}$ with respect to $\alpha$ (explicit expressions can be found in Ref. 12), and $\mathscr{N}(\varepsilon)$ is the density of states (DOS) of freely hopping electrons. The dimension dependence resides solely in $\mathscr{N}(\varepsilon)$, and, since this DOS applies to a noninteracting system, I will be able to derive results for arbitrary dimension in Sec. IV (see also the Appendix). Note that the above set of equations reduces to the familiar antiferromagnetic or spin-density-wave (SDW) solution in the HFA if $q_{s}=1 .^{3}$ In that case, one has one selfconsistency equation (2), which is the gap equation with $\bar{\lambda}$ as the gap parameter $\Delta\left(\equiv U m_{s}\right)$, instead of three selfconsistency equations (2)-(4).

The energy of the AF state (per site) and the spin stiffness $\rho_{s}$ are given by

$$
\begin{gathered}
e_{\mathrm{AF}}=-2 q_{s} \int_{\bar{\mu}}^{\infty} d \varepsilon \mathscr{N}(\varepsilon) \sqrt{\varepsilon^{2}+\bar{\lambda}^{-2}}+U d^{2}+\bar{\lambda} q_{s} m_{s}, \\
\rho_{s}=\frac{q_{s}}{4} \int_{\bar{\mu}}^{\infty} d \varepsilon \frac{\mathscr{N}(\varepsilon) \varepsilon^{2}}{\left(\varepsilon^{2}+\bar{\lambda}^{-2}\right)^{1 / 2}}-\frac{z_{+}^{2} z_{-}^{2}}{q_{s}} \int_{\bar{\mu}}^{\infty} d \varepsilon \frac{\mathscr{N}_{\mathbf{v}}(\varepsilon) \varepsilon^{2}}{\left(\varepsilon^{2}+\lambda^{-2}\right)^{3 / 2}} .
\end{gathered}
$$

$\mathscr{N}_{\mathbf{v}}(\varepsilon)$ is the weighted DOS, which, as well as the combination $z_{+} z_{-}$, is specified in Ref. 12. I will not repeat the expressions here, since I will now restrict to half-filling $(\bar{\mu}=0$, $n=1$ ), for which case $z_{+} z_{-}$equals zero. For half-filling, the band renormalization reduces to

$$
q_{s}\left(1, m_{s}, d\right)=\frac{2 d^{2}\left[\sqrt{1-2 d^{2}+m_{s}}+\sqrt{1-2 d^{2}-m_{s}}\right]^{2}}{1-m_{s}^{2}} .
$$

\section{LARGE- $U$ EXPANSION IN TERMS OF MOMENTS}

The above set of equations (2)-(5) can be solved numerically if the DOS for freely hopping electrons is given. For instance, in two dimensions $\mathscr{N}(\varepsilon)$ is known analytically, and numerical solutions to (2)-(5) were obtained in Ref.
12. Now I will derive solutions to (2)-(5) for $n=1$ in the form of a systematic series expansion in $1 / U$. This expansion is derived in two steps: (i) From the explicit form of the equations above (or from numerical solutions) it can be seen that large $U$ corresponds to large $\bar{\lambda}$. Therefore, I first make a large $-\bar{\lambda}$ expansion for all quantities of interest. Subsequently, (ii) the $\bar{\lambda}$ expansion for $U$ [following from Eq. (4)] is inverted and substituted in the large- $\bar{\lambda}$ expansion for all relevant quantities obtained before. In this way, one obtains the desired large- $U$ expansion.

By expanding the square root in the denominator of the integrals in $(2)-(5)$ for $\bar{\lambda} \gg \varepsilon$, one obtains a large- $\bar{\lambda}$ expansion in terms of moments $M_{n}$ of the DOS:

$$
M_{n}=\int_{-\infty}^{\infty} d \varepsilon \mathscr{N}(\varepsilon) \varepsilon^{n}
$$

After some work one arrives at the following expansions in $q \equiv 1 / \bar{\lambda}^{-}:^{13}$

$$
\begin{aligned}
& m_{s}=1-\frac{1}{2} M_{2} q^{2}+\frac{3}{8} M_{4} q^{4}-\frac{5}{16} M_{6} q^{6}+\frac{35}{128} M_{8} q^{8}+O\left(q^{10}\right), \\
& d=\frac{1}{2} \sqrt{M_{2}} q-\frac{4 M_{2}^{2}+3 M_{4}}{16 \sqrt{M_{2}}} q^{3}+\frac{\sqrt{M_{2}}}{16}\left[M_{2}^{2}+\frac{13}{2} M_{4}-\frac{9 M_{4}^{2}}{16 M_{2}^{2}}\right. \\
& \left.+\frac{5 M_{6}}{2 M_{2}}\right] q^{5}-\frac{1}{2048 M_{2}^{5 / 2}}\left[192 M_{2}^{6}+368 M_{2}^{4} M_{4}\right. \\
& +364 M_{2}^{2} M_{4}^{2}+27 M_{4}^{3}+736 M_{2}^{3} M_{6}-120 M_{2} M_{4} M_{6} \\
& \left.+280 M_{2}^{2} M_{8}\right] q^{7}+O\left(q^{9}\right), \\
& q_{s}=1-\frac{M_{2}}{4} q^{2}-\left[\frac{M_{2}^{2}-7 M_{4}}{16}\right] q^{4} \\
& -\left[\frac{9 M_{2}^{3}-10 M_{2} M_{4}+12 M_{4}^{2} / M_{2}+26 M_{6}}{64}\right] q^{6}+O\left(q^{8}\right), \\
& U=\frac{2}{q}+\frac{5}{2} M_{2} q+\left[\frac{11 M_{2}^{2}-21 M_{4}}{8}\right] q^{3}+O\left(q^{5}\right) \\
& e_{\mathrm{AF}}=-\frac{1}{2} M_{2} q+\left[\frac{3 M_{2}^{2}+M_{4}}{8}\right] q^{3}+\left[\frac{M_{2}^{3}-22 M_{2} M_{4}-2 M_{6}}{32}\right] q^{5} \\
& +O\left(q^{7}\right) \\
& \rho_{s}=\frac{M_{2}}{8} q-\left[\frac{M_{2}^{2}+2 M_{4}}{32}\right] q^{3}+\left[\frac{-M_{2}^{3}+9 M_{2} M_{4}+6 M_{6}}{128}\right] q^{5} \\
& -\left[\frac{9 M_{2}^{4}-12 M_{2}^{2} M_{4}+26 M_{4}^{2}+32 M_{2} M_{6}+20 M_{8}}{512}\right] q^{7} \\
& +O\left(q^{9}\right) .
\end{aligned}
$$

For notational convenience the hopping integral $t$ is taken equal to 1 in this section. For $m_{s}, d$, and $q_{s}$, I have listed precisely the number of terms that is needed in order to obtain the number of terms (three) that is listed for $U$. For $e_{\mathrm{AF}}$ and $\rho_{s}$, terms are given that can be found using the listed 
terms for $m_{s}, d, q_{s}$, and $U$. Note that this implies that, to obtain the leading order in $q$ for $U$, one already needs three terms for $m_{s}$ and two terms for $d$.

Inverting the expansion for $U$ gives

$$
q=2 p+10 M_{2} p^{3}+\left(122 M_{2}^{2}-42 M_{4}\right) p^{5}+O\left(p^{7}\right),
$$

where I have introduced the notation $p \equiv 1 / U$. Substituting this into the $q$ expansions, one obtains the following large- $U$ expansions:

$$
\begin{aligned}
m_{s}=1 & -2 M_{2} p^{2}-\left[20 M_{2}^{2}-6 M_{4}\right] p^{4}-\left[294 M_{2}^{3}-204 M_{2} M_{4}\right. \\
+ & \left.20 M_{6}\right] p^{6}+O\left(p^{8}\right), \\
d^{2}= & M_{2} p^{2}+\left[6 M_{2}^{2}-3 M_{4}\right] p^{4}+\left[75 M_{2}^{3}-70 M_{2} M_{4}\right. \\
& \left.+10 M_{6}\right] p^{6}+O\left(p^{8}\right), \\
q_{s}= & 1-M_{2} p^{2}-\left[11 M_{2}^{2}-7 M_{4}\right] p^{4}-\left[176 M_{2}^{3}-192 M_{2} M_{4}\right. \\
+ & \left.12 M_{4}^{2} / M_{2}+26 M_{6}\right] p^{6}+O\left(p^{8}\right), \\
e_{\mathrm{AF}}= & -M_{2} p-\left[2 M_{2}^{2}-M_{4}\right] p^{3}-\left[15 M_{2}^{3}-14 M_{2} M_{4}\right. \\
& \left.+2 M_{6}\right] p^{5}+O\left(p^{7}\right), \\
& M_{2} p+\frac{1}{2}\left[2 M_{2}^{2}-M_{4}\right] p^{3}+\frac{3}{4}\left[15 M_{2}^{3}-14 M_{2} M_{4}\right. \\
\rho_{s}= & \left.\quad+2 M_{6}\right] p^{5}+O\left(p^{7}\right) .
\end{aligned}
$$

A consistency check on the above results is that the following general identity still holds for the expansions above:

$$
\frac{\partial e_{\mathrm{AF}}}{\partial U}=d^{2}
$$

Because in the SBMF at half-filling the spin stiffness is given by $-\frac{1}{8}$ times the average kinetic energy, ${ }^{12}$ we also have

$$
\rho_{s}=-\frac{1}{8}\left(e_{\mathrm{AF}}-U d^{2}\right) .
$$

Combining the latter formula with (24), it immediately follows that the expansion for $\rho_{s}$ follows directly from the expansion for $e_{\mathrm{AF}}$, as is also seen in the way the expansions are written above.

It is of some interest to compare the present result with results from the HFA and other expressions in the literature. It is far easier to derive the corresponding expression to (22) in the HFA since there is only one consistency equation (the gap equation) instead of three, and there is no bandrenormalization function $q_{s}$ which requires perturbative expanding. Following the same procedures as for the SBMF, for the HFA I find

$$
\begin{aligned}
e_{\mathrm{AF}}= & -M_{2} p-\left[M_{2}^{2}-M_{4}\right] p^{3}-\left[4 M_{2}^{3}-6 M_{2} M_{4}+2 M_{6}\right] p^{5} \\
& +O\left(p^{7}\right) .
\end{aligned}
$$

Comparing with (22), one sees that the HFA and SBMF give the same coefficient for the leading-order contribution, but that there are differences for the higher-order contributions. Note also that for higher-order contributions the term in the coefficient involving the highest moment agrees between the HFA and SBMF. Since the moments are always positive, it is also clear that for large $U$ the energy in the SBMF approximation is always lower than in the HFA.

In dimensions $D=2$ and 3 (square and simple cubic lattices), the results can also be compared with a large- $U$ expansion by Takahashi. ${ }^{14} \mathrm{He}$ derived a rigorous expansion (i.e., without making approximations) for the half-filled Hubbard model in terms of spin-correlation functions of the $s=$ $\frac{1}{2}$ Heisenberg model. These correlation functions are then evaluated using the linear-spin-wave (LSW) approximation. Below, the results of the latter approach are compared to those of the HFA and SBMF for $D=2$ and 3 (the necessary moments are easily evaluated and may for instance be found in the Appendix):

$$
\begin{array}{ccc} 
& D=2 & D=3 \\
\text { HFA } & -4 p+20 p^{3}-192 p^{5}+\cdots, & -6 p+54 p^{3}-1344 p^{5}+\cdots \\
\text { SBMF } & -4 p+4 p^{3}+256 p^{5}+\cdots, & -6 p+18 p^{3}+600 p^{5}+\cdots \\
\text { LSW } & -4.63 p+34.6 p^{3}+\cdots, & -6.58 p+65.6 p^{3}+\cdots
\end{array}
$$

It is clear that for large enough $U$ the LSW expansion will give the lower energy. The leading-order coefficient is proportional to the ground-state energy of the $s=\frac{1}{2}$ Heisenberg antiferromagnet, which is known to be very well approximated by the LSW. ${ }^{15,16}$ The HFA and SBMF just reproduce the mean-field result for the leading-order coefficient. In view of the fact that the SBMF is an improvement over the HFA, it is somewhat remarkable that the HFA results for the next-to-leading order resemble the LSW results more than the SBMF results do. Also, the different sign of the coefficient of $p^{5}$ between the HFA and SBMF is notable. Further discussion of the range of validity of the expansion is given below.
To end this section a quantitative discussion of the large- $U$ expansion is also given. In Table I, the ground-state energy of the AF phase at half-filling, as obtained from the large- $U$ expansion within the $\operatorname{SBMF}\left(e_{\mathrm{AF}}^{U_{\text {exp }}}\right)$, is compared to the "exact SBMF" result $\left[e_{\mathrm{AF}}^{\mathrm{SBMF}}\right.$, found by solving (2) $-(5)$ numerically] and to variational Monte Carlo (VMC) results using an (antiferromagnetic) Gutzwiller wave function $\left(e_{\mathrm{AF}}^{\mathrm{GWMC}}\right.$, from Ref. 17$)$ for $D=2$. For $D=3$, only $e_{\mathrm{AF}}^{U_{\text {exp }}}$ and $e_{\mathrm{AF}}^{\mathrm{GWVMC}}$ are compared. Note that the large- $U$ expansion approximates the "exact $\mathrm{SBMF}$ " result very well for $U / t \geqslant 7$; it is expected that similar agreement is found for $D=3$, where it is more involved to compute $e_{\mathrm{AF}}^{\mathrm{SBMF}}$ since $\mathscr{N}(\varepsilon)$ is not a known analytical function as it is for $D=2 .{ }^{18}$ This is consis- 
TABLE I. Numerical results at $D=2$ and 3 for the ground-state energy of the antiferromagnetic phase at half-filling. $e_{\mathrm{AF}}^{U_{\mathrm{exp}}}$ is evaluated using the large- $U$ expansion (22), and $e_{\mathrm{AF}}^{\mathrm{SBMF}}$ is obtained from (8) after solving (2)-(5). The GWVMC results are taken from Ref. 17, and were obtained on $20 \times 20$ and $6 \times 6 \times 6$ lattices for $D=2$ and 3, respectively. Between brackets the statistical error in the last digit is given.

\begin{tabular}{|c|c|c|c|c|c|}
\hline \multirow[b]{2}{*}{$U$} & \multicolumn{3}{|c|}{$D=2$} & \multicolumn{2}{|c|}{$D=3$} \\
\hline & $-e_{\mathrm{AF}}^{\mathrm{SBMF}}$ & $-e_{\mathrm{AF}}^{U_{\text {exp }}}$ & $-e_{\mathrm{AF}}^{\mathrm{GWVMC}}$ & $-e_{\mathrm{AF}}^{U_{\exp }}$ & $-e_{\mathrm{AF}}^{\mathrm{GWVMC}}$ \\
\hline 6 & 0.623639 & 0.615226 & $0.629(3)$ & 0.8395 & $0.886(5)$ \\
\hline 8 & 0.485104 & 0.484375 & $0.493(3)$ & 0.6965 & $0.704(7)$ \\
\hline 10 & 0.393528 & 0.393440 & $0.401(4)$ & 0.5760 & $0.579(6)$ \\
\hline 12 & 0.330002 & 0.329990 & $0.336(5)$ & 0.4872 & $0.491(7)$ \\
\hline 16 & 0.248782 & 0.248779 & & 0.3700 & \\
\hline 20 & 0.199420 & 0.199420 & & 0.2976 & \\
\hline
\end{tabular}

tent with the fact that from the coefficients in the explicit expansions for $D=2$ and 3 , formula (27), one would estimate the series to converge well for $U / t \geqslant 8$ and $U / t \geqslant 6$ for two and three dimensions, respectively. The SBMF is equivalent to the Gutzwiller approximation to the Gutzwiller wave function, ${ }^{9}$ and this approximation becomes exact in the limit of an infinite number of spatial dimensions. ${ }^{19,20}$ From the comparison with the GWVMC results it can be seen that the approximation is already quite good for $D=2$. In view of the facts that for $D=3$ a very small lattice was used in the GWVMC calculations, and a larger lattice will even raise the energy somewhat (the effect will be larger for smaller $U$ ), the agreement for $D=3$ can even be called excellent. Thus the clear advantage of the large- $U$ expansion is that it can give results that are, for $U / t \geqslant 8$, within $1 \%$ of the "exact SBMF" and GWVMC calculations (which especially for $D=3$ are much more involved and computationally demanding) by means of the simple formula (22).

\section{LARGE- $U$ EXPANSION FOR ARBITRARY DIMENSION}

In the Appendix, it is shown that the moments $M_{n}$ can be obtained as a function of dimension $D$ [formula (A9)]. If one substitutes these expressions into (22) and (26), one obtains a simultaneous $1 / U$ and $1 / D$ expansion for the energy of the AF phase:

$$
\begin{aligned}
\operatorname{SBMF} e_{\mathrm{AF}}= & -\frac{2 D t^{2}}{U}+\left(1-\frac{3}{2 D}\right) \frac{4 D^{2} t^{4}}{U^{3}} \\
& +\left(-3+\frac{24}{D}-\frac{20}{D^{2}}\right) \frac{8 D^{3} t^{6}}{U^{5}}+\cdots, \\
\mathrm{HFA} e_{\mathrm{AF}}= & -\frac{2 D t^{2}}{U}+\left(2-\frac{3}{2 D}\right) \frac{4 D^{2} t^{4}}{U^{3}} \\
& +\left(-16+\frac{36}{D}-\frac{20}{D^{2}}\right) \frac{8 D^{3} t^{6}}{U^{5}}+\cdots,
\end{aligned}
$$

where I have again included the hopping parameter $t$. The above formulas are well suited to discuss the limit of an infinite number of dimensions. This is of interest since, as noted above, the SBMF is equivalent to the Gutzwiller approximation to the Gutzwiller wave function, an approximation which becomes exact in the limit of an infinite number of spatial dimensions. Thus the present expansion allows us to scrutinize the approximation explicitly for lower dimension. In order for the limit $D \rightarrow \infty$ to be meaningful, one has to introduce a scaled hopping parameter $t^{*}=t \sqrt{2 D} .{ }^{19}$ In terms of this scaled parameter the above SBMF large- $U$ expansion assumes the form

$$
\begin{aligned}
\frac{e_{\mathrm{AF}}}{t^{*}}= & -\frac{t^{*}}{U}+\left(1-\frac{3}{2 D}\right)\left(\frac{t^{*}}{U}\right)^{3}+\left(-3+\frac{24}{D}-\frac{20}{D^{2}}\right) \\
& \times\left(\frac{t^{*}}{U}\right)^{5}+\cdots
\end{aligned}
$$

In Table II, I evaluate (30) for $D=2,3,4$, and $\infty$. From Table II, one sees that for scaled interaction $U^{*} \geqslant 4$ (which corresponds to $U / t=8$ for $D=2$ ) the result for $D=2$ and the infinite-dimensional result already coincide remarkably well. The simultaneous large- $U$, large- $D$ expansion shows in detail how well the Gutzwiller approximation, and therefore the SBMF, reproduces the Gutzwiller wave function for the various dimensions. It would have been interesting to compare the coefficients in the present results with those obtained by Gebhard in his $1 / D$ expansion. ${ }^{20}$ Unfortunately, a direct comparison is not meaningful, since Gebhard only considers the paramagentic Gutzwiller wave function and does not treat the antiferromagnetic phase at half-filling in a $1 / D$ expansion.

\section{DISCUSSION AND CONCLUSIONS}

There is no fundamental reason why the large- $U$ expansion given here cannot be extended to higher orders in $t / U$.

TABLE II. Scaled ground-state energy of the AF phase at halffilling $e_{\mathrm{AF}}^{*} \equiv e_{\mathrm{AF}} / t^{*}$ within the SBMF as a function of scaled interaction $U^{*} \equiv U / t^{*}$ for various dimensions $D$ (with $t^{*}=t \sqrt{2 D}$ ).

\begin{tabular}{rcccc}
\hline \hline$U^{*}$ & $-e_{\mathrm{AF}}^{*}(D=2)$ & $-e_{\mathrm{AF}}^{*}(D=3)$ & $-e_{\mathrm{AF}}^{*}(D=4)$ & $-e_{\mathrm{AF}}^{*}(D=\infty)$ \\
\hline 2 & 0.34375 & 0.35069 & 0.36719 & 0.46875 \\
4 & 0.24219 & 0.23947 & 0.23853 & 0.23730 \\
8 & 0.12439 & 0.12394 & 0.12373 & 0.12314 \\
12 & 0.08317 & 0.08303 & 0.08296 & 0.08277 \\
16 & 0.06244 & 0.06238 & 0.06235 & 0.06226 \\
20 & 0.04997 & 0.04994 & 0.04992 & 0.04988 \\
\hline \hline
\end{tabular}


With a little more effort [especially the coefficients in the large- $\bar{\lambda}$ expansion of $d$ in formula (13) require some care] more terms can be obtained. However, as already remarked in Sec. III, the present series reproduces well the "exact SBMF" result for $U / t \geqslant 7$ in two dimensions, and this agreement is expected to be even better in three dimensions. Also, for smaller values of $U / t$ the contribution from the $(t / U)^{5}$ term becomes larger than that from the $(t / U)^{3}$ term, which is an indication that the intrinsic radius of convergence of the series is close to $U / t=7$. Therefore, extending the series cannot be considered very useful.

A more interesting extension of the present series is to go off half-filling, i.e., $n \neq 1$. Again there is no fundamental reason preventing this, but now the task is quite a bit more laborious for a number of reasons. Not only are we dealing with the case $\bar{\mu} \neq 0$, and, consequently, partial moments are required [see (11)], which can only be computed numerically, but also the expression for the band renormalization $q_{s}$, formulas (6) and (7), needs to be expanded for $n \neq 1$, which implies that nonrational coefficients in the expansion in terms of (partial) moments appear. Furthermore, in formula (9) for $\rho_{s}$ the term with the combination $z_{+} z_{-}$ also needs to be taken into account and perturbatively expanded. If similar large- $U$ expansions are possible for more complicated phases, like spirals or domain walls, this would allow for a study of aspects of the phase diagram off halffilling.

In conclusion, a large- $U$ expansion is derived for the antiferromagnetic phase of the Hubbard model within the framework of the slave-boson mean-field approximation. Even though such an expansion constitutes in a sense an approximation of an approximation, the resulting analytic expression is capable of reproducing very well results of elaborate Monte Carlo calculations. Furthermore, the expansion allows for an explicit study of the limit of a large number of spatial dimensions, since the occurring moments of the noninteracting density of states are obtained for arbitrary dimension.

Note added in proof. Recently, I was informed that a strong-coupling expansion at and near half-filling for two dimensions is discussed in B. Möller, K. Doll, and R. Frésard, J. Phys. Condens. Matter 5, 4847 (1993); K. Doll, Diploma thesis, Karlsruhe, 1992.

\section{ACKNOWLEDGMENTS}

I acknowledge useful discussions with P. G. J. van Dongen, F. Gebhard, and J. M. J. van Leeuwen on the work reported here, as well as comments by D. P. Aalberts on an earlier version of the paper.

\section{APPENDIX}

In this appendix some general expressions are derived for the moments $M_{n}$ of the density of states $\mathscr{N}(\varepsilon)$ of freely hopping electrons on hypercubic lattices:

$$
\begin{aligned}
M_{n} & =\int_{-\infty}^{\infty} d \varepsilon \mathscr{N}(\varepsilon) \varepsilon^{n} \\
& =\frac{(2 t)^{n}}{(2 \pi)^{D}} \int_{-\pi}^{\pi} \cdots \int_{-\pi}^{\pi} d k_{1} \ldots d k_{D}\left[\cos k_{1}+\cdots+\cos k_{D}\right]^{n} .
\end{aligned}
$$

Although such moments are frequently used and calculated in the literature, I have never encountered the general analytic expressions and simple formulas to be given below. First I will derive simple expressions for all moments in the lower dimensions $D=1,2$, and 3. Then I will derive expressions for the lower moments in arbitrary dimension $D$.

By employing the multinomial generalization of the binomial, it is possible to further work out (A1):

$$
M_{n}=(2 t)^{n} \sum_{n_{1}, \ldots, n_{D}=0}^{n}{ }^{\prime} \frac{n !}{n_{1} ! \ldots n_{D} !} q_{n_{1} \ldots q_{n_{D}}},
$$

where the prime on the sum denotes the restriction $n_{1}+\cdots+n_{D}=n$ and

$$
q_{m}=\frac{1}{2 \pi} \int_{0}^{2 \pi} d k \cos ^{m}(k)= \begin{cases}\frac{(m-1) ! !}{m ! !} & m \text { even } \\ 0 & m \text { odd. }\end{cases}
$$

From this general form it is clear that all moments with $n$ odd are zero for any dimension. All formulas below will be for moments with $n$ even. For $D=1$ the restriction allows for only one term in the sum, and the result is trivial:

$$
M_{n}=(2 t)^{n} \frac{(n-1) ! !}{n ! !}, \quad D=1 .
$$

A more remarkable result is that the moments for $D=2$ are exactly the squares of the moments for $D=1$ (if $t$ is put equal to 1$)$ :

$$
M_{n}=t^{n}\left[2^{n} \frac{(n-1) ! !}{n ! !}\right]^{2}, \quad D=2 .
$$

The proof of (A5) follows directly from (A2) by some manipulations with the factorials, and employing the identity ${ }^{21}$

$$
\sum_{n=0}^{N}\left(\begin{array}{l}
N \\
n
\end{array}\right)^{2}=\left(\begin{array}{c}
2 N \\
N
\end{array}\right)
$$

For $D=3$ I have not been able to obtain an explicit expression for $M_{n}$, but the triple sum in (A2) can be reduced to one simple, unrestricted sum. Using the relations $(m-1) ! ! / m !=1 / m ! !=2^{-m / 2} /(m / 2) !(m$ even $)$, one can rewrite (A2) (for $n$ even) as

$$
M_{n}=n ! \sum_{u, v, w=0}^{n / 2} \frac{1}{(u !)^{2}(v !)^{2}(w !)^{2}},
$$

where the prime indicates the constraint $u+v+w=n / 2$. This expression was already given by Brinkman and Rice for the moments of the density of states of one hole in a ferromagnetic background. ${ }^{22}$ It can, however, be simply reduced to a single sum by incorporating the constraint and a subsequent application of (A6). The result is 


$$
M_{n}=n ! \sum_{u=0}^{n / 2} \frac{(n-2 u) !}{(u !)^{2}[(n / 2-u) !]^{4}}, \quad D=3 .
$$

Formulas (A5) and (A8) make for a much easier evaluation of the moments than is usually found in the literature: e.g., in Ref. 23 moments up to $n=22$ and 16 are computed for $D=2$ and 3 , respectively, using the method of counting the number of paths that return to the origin after $n$ steps. I note that also finding an explicit expression for $D=3$ may be possible on account of the fact that the moments for $D=3$ always contain the moments for $D=1$ as a factor.

The general expression (A2) shows that finding the terms contributing to $M_{n}$ amounts to finding all the combinations of $D$ even numbers (including zero) that add up to $n$. For the lower moments the number of possibilities is not large and can be expressed in terms of $D$ using combinatorial arguments. I have found the following results:

$$
\begin{gathered}
M_{2}=2 D, \\
M_{4}=6 D(2 D-1), \\
M_{6}=20 D\left(6 D^{2}-9 D+4\right), \\
M_{8}=70 D\left(24 D^{3}-72 D^{2}+82 D-33\right), \\
M_{10}=252 D\left(14400 D^{4}-143400 D^{3}+501050 D^{2}\right. \\
-715225 D+343176) .
\end{gathered}
$$

and most quantum Monte Carlo work is for two dimensions (where $W=8 t$ ) and $U / t=4$.

${ }^{12}$ P. J. H. Denteneer and M. Blaauboer, J. Phys. Condens. Matter 7, 151 (1995); 7, 2377(E) (1995).

${ }^{13}$ The expansion for $m_{s}$ follows directly from (2), and the expansion for $d$ is first written with general coefficients which are then determined by the fact that (3) must be satisfied (this requires an expansion of $\left.\left[q_{s}\right]_{m_{s}}\right)$. The expansions for $U, e_{\mathrm{AF}}$, and $\rho_{s}$ then follow from those for $m_{s}$ and $d$ (they require expansions of $\left[q_{s}\right] d$ and $\left.q_{s}\right)$.

${ }^{14}$ M. Takahashi, J. Phys. C 10, 1289 (1977).

${ }^{15}$ P. W. Anderson, Phys. Rev. 86, 697 (1952)

${ }^{16}$ D. C. Mattis, The Theory of Magnetism (Harper and Row, New York, 1982).

${ }^{17}$ H. Yokoyama and H. Shiba, J. Phys. Soc. Jpn. 56, 3582 (1987).

${ }^{18}$ The "exact SBMF" result for $D=3$ is obtained by H. Hasegawa, Phys. Rev. B 41, 9168 (1990); it is compared to the GWVMC result of Ref. 17 only graphically, but the quantitative agreement appears good.

${ }^{19}$ W. Metzner and D. Vollhardt, Phys. Rev. Lett. 62, 324 (1989).

${ }^{20}$ F. Gebhard, Phys. Rev. B 41, 9452 (1990).

${ }^{21}$ I. S. Gradsteyn and I. M. Ryzhik, Table of Integrals, Series, and Products (Academic, New York, 1980), formula 0.157.

${ }^{22}$ W. F. Brinkman and T. M. Rice, Phys. Rev. B 2, 1324 (1970).

${ }^{23}$ D. F. B. ten Haaf, P. W. Brouwer, P. J. H. Denteneer, and J. M. J. van Leeuwen, Phys. Rev. B 51, 353 (1995). 